\title{
ÜBER EINIGE PROBLEME BEIM WISSENSCHAFTLICHEN UMGANG MIT DEM BEGRIFF DES TYPISCHEN UND ÜBER DEN VERSUCH, IHN AUF SINNVOLLE WEISE DURCH EINEN ANDEREN ZU ERSETZEN
}

\begin{abstract}
Ekkehard Jost
Zunächst einmal möchte ich mich sehr herzlich dafür bedanken, dass mir die Gelegenheit gegeben wird, hier an meiner alten Wirkungsstätte, an der ich 30 Jahre lang aktiv sein durfte, mal wieder in Erscheinung zu treten.

Als mich vor einiger Zeit mein Freund und Kollege Thomas Phleps zu dieser Tagung einlud und mir den Titel derselben nannte, bekam ich einen Schreck und signalisierte ihm wenig später, dass mich diese Vorgabe in tiefes Grübeln geworfen hätte. »Typisch deutsch«, fragte ich mich und ihn, zielt das überhaupt auf eine Fragestellung, die einen wie auch immer gearteten wissenschaftlichen Zugriff erlaubt? Oder landen wir damit nicht vielmehr bei einem eher feuilletonistischen Ansatz oder gar bei einem jener StandardKalauer nach dem Schema »Treffen sich ein Brite, ein Italiener, ein Franzose und ein Deutscher in einem Rettungsboot « - oder wo auch immer?

Ich habe einmal - in grauer Vorzeit - an der Universität Hamburg bei dem seinerzeit verdientermaßen sehr renommierten Wiener Psychologen Peter R. Hofstätter studiert - wie ich glaube, mit großem Gewinn. Hofstätters Einführung in die Sozialpsychologie (1966) war damals für unseren kleinen Kreis jugendlicher Studenten, die bei Hans Peter Reinecke an der Hamburger Uni »Systematische Musikwissenschaft « studierten, so etwas wie ein »Sesam, öffne Dich!« - hinein in die wissenschaftliche Forschung. Von Hofstätter erfuhren wir, dass Wortgebilde wie »typisch Mann«, »typisch Beamter «, »typisch deutsch«, »typisch soundsoetwas« in das Begriffsfeld der Stereotype, Vorurteile, Klischeebildungen usw. gehören, und damit primär in einen Zuständigkeitsbereich der Sozialpsychologie, der einem musikologisch-analytischen Zugriff im engeren Sinne nur schwer - wenn überhaupt zugänglich ist, sondern der eher das Terrain des Feuilletons und sonstiger spekulativer Denkbereiche betrifft.
\end{abstract}


Daraus folgt für mich hier und heute: ich werde unser Tagungsthema zunächst einmal aus methodologischer und insbesondere sozialpsychologischer Perspektive beleuchten und dabei zugleich versuchen, auf die bisher zu Tage geförderten Forschungsergebnisse zum Thema überzublenden. In einem zweiten Schritt werde ich versuchen, am Beispiel der so genannten Europäischen Jazz-Emanzipation der 1960er und 1970er Jahre darzustellen, wie und warum es unter bestimmten Bedingungen auch in der Musik im Allgemeinen und im Jazz im Speziellen zu regional oder national typischen Erscheinungsformen kommen kann, die eine (vorsichtige) Verwendung des Begriffs des »Typischen« rechtfertigen könnten. Ich bin mir natürlich dessen bewusst, dass ich mit meiner Konzentration auf den Jazz hier im Arbeitskreis Studium Populärer Musik e.V. (ASPM) auf dem falschen Dampfer reise, denn der Jazz und noch dazu der zeitgenössische Jazz sind bekanntlich alles andere als populär. Aber erstens kenne ich mich auf dem Terrain des Jazz wesentlich besser aus als im Feld der so genannten Populären Musik (was auch immer das im einzelnen sein mag) und zweitens gehe ich davon aus, dass ich Ihnen einen solchen Ausflug in etwas entferntere musikalische Welten durchaus einmal zumuten darf.

Mein Hinweis auf die Bedeutung des »Besser-Auskennens « ist übrigens nicht unbedingt zu verstehen als der Ausdruck des Kokettierens mit dem Zustand relativer Ahnungslosigkeit im Bereich der so genannten populären Musik, sondern vielmehr als ein Hinweis auf ein gravierendes methodisches Problem: Attribute wie jene des typisch Deutschen, Britischen oder Französischen zielen im Allgemeinen auf etwas Exklusives, Einmaliges. Eine bestimmte Eigenschaft, Gewohnheit, oder Fähigkeit einer bestimmten Gruppe von Menschen, einer Region oder Nation kann als typisch für diese nur dann angesehen werden, wenn das betreffende Merkmal für andere Gruppen von Menschen, für andere Regionen oder andere Nationen keine oder nur eine sehr geringe Rolle spielt. Es bedarf - wie es auf Neudeutsch heißt - eines »Alleinstellungsmerkmals«. Das »Tanzen im Dreiertakt und in Sepplhosen und mit einem Hütchen auf dem Kopf « kann - mit einiger Berechtigung nur dann als »typisch bayerisch « klassifiziert werden, wenn es in anderen Regionen oder Ländern keine oder lediglich eine periphere Rolle spielt.

Aus der Bedeutung der relativen Einmaligkeit von Merkmalen und deren quantitativem Gewicht innerhalb einer bestimmten Population folgt eine sehr wichtige Voraussetzung für unsere Forschungspraxis: Aussagen über ein bestimmtes Phänomen, das als typisch für eine soziale Gruppe, für einen Berufsstand, eine Region oder ein Volk usw. identifiziert werden kann, erfordern - genau genommen - ein globales Wissen über dieses Phänomen in anderen Gruppen, Nationen, Kulturen usw. Und damit kommen ich zurück 
auf mein ganz persönliches Problem mit dem mir vom Kollegen Phleps präsentierten Thema: Antworten auf die Frage, was »typisch deutsch « sei in der populären Musik, kann ich nur leisten, wenn ich die populären Musiken anderer Völker, Regionen, Ethnien usw. genau so gut kenne wie die, welche mich unmittelbar umgibt. Und das ist, zugegebenermaßen bei einem Großteil der Musik, die im Zentrum der Arbeit unseres »Arbeitskreises Studium Populäre Musik« steht, bei mir leider nicht unbedingt der Fall. Insofern könnte ich es Ihnen nicht verübeln, wenn Sie mich bei Gelegenheit aus unserem Verein rauswerfen möchten, denn die Musik, mit der ich mich seit einigen Jahrzehnten auf wissenschaftlicher Ebene schwerpunktmäßig beschäftige und die ich auch selbst praktiziere, ist nun wirklich alles andere als populär. Andererseits aber ist es in unserer scientific community bekanntlich durchaus unüblich, jemanden aus einer wissenschaftlichen Gesellschaft nur deshalb auszuschließen, weil er oder sie von dem betreffenden Gegenstand keine Ahnung hat. Insofern fühle ich mich beim ASPM immer noch einigermaßen wohl und sicher.

Spaß beiseite! - Die Tatsache, dass ich mich schwerpunktmäßig mit einer nicht-populären Musik befasse und daher in gewisser Weise als ASPMOutcast zu gelten habe, wird mich allerdings nicht von dem Versuch abhalten, Ihnen hier - in der gebotenen Kürze - am Beispiel jener unpopulären Musik namens »zeitgenössischer Jazz « zu demonstrieren, wie man - unter Umständen - jener fragwürdigen Kategorie des »Typischen« dennoch einigermaßen erfolgreich zu Leibe rücken kann.

Zunächst aber erst einmal die Frage: Was gibt die vorhandene oder jedenfalls zugängliche Literatur zum Thema »Typisch deutsch« eigentlich her? Ich kann mich kurz fassen: Sehr wenig, oder - genauer gesagt - in wissenschaftlicher Hinsicht so gut wie nichts Brauchbares. Die einzige im weitesten Sinne wissenschaftlich zu nennende Publikation erschien im Jahr 2000 unter dem Titel Typisch deutsch. Wie deutsch sind die Deutschen und stammt von dem Tübinger Kulturwissenschaftler Hermann Bausinger. ${ }^{1}$ Bevor ich auf Bausingers Schrift ein wenig detaillierter eingehe, will ich kurz die übrige, von mir gesichtete Literatur unter die Lupe nehmen. Es handelt sich dabei erstens um das schwergewichtige und 560 Seiten umfassende Werk des Autoren-Teams Thea Dorn und Richard Wagner Die deutsche Seele (2011), zweitens die Arbeit des Anglisten Hans-Dieter Gelfert über Was ist deutsch? Wie die Deutschen wurden, was sie sind (2005), drittens die Schrift Feierabend. Eine Reise in die deutsche Seele von Vanna Vannuccini und

1 Bausinger wurde meines Wissens auch zu dieser Tagung eingeladen, konnte jedoch nicht kommen, was ich sehr bedaure. 
Francesca Predazzi (2013) und zu guter Letzt Die Legende vom typisch Deutschen. Eine Kultur im Spiegel der Franzosen von Béatrice Durand (2004).

Allen vier Publikationen gemeinsam ist, dass sie sich gut lesen und viele interessante Fakten und Gedanken enthalten - und dass sie sich durchweg im Bereich des Feuilletons und des Spekulativen bewegen. Die immer wieder gleichen Themen kreisen um Begriffe wie Heimat, Gemütlichkeit, Ordnung, Pünktlichkeit, Tüchtigkeit, Fleiß, Gründlichkeit, Treu und Redlichkeit, Innigkeit, Weltschmerz, Wald, Weihnacht und so weiter. Von Musik ist gelegentlich auch die Rede, aber wenn, dann grundsätzlich von klassischer oder so genannter »Ernster « Musik, wobei die in diesem Zusammenhang aufgetischten Banalitäten musikologischen Standards allerdings nur sehr selten gerecht werden.

Um auf Bausinger zurückzukommen: Er relativiert den wissenschaftlichen Charakter seiner Fragestellung von vornherein selbst, indem er - unter der Überschrift »Sinn und Unsinn der Typisierung « feststellt: Der Begriff des Typischen sei keine wissenschaftliche Kategorie, sondern eine Zuschreibung. Ich stimme zu. Wobei zu ergänzen wäre: einer Zuschreibung, die auf Meinungen basiert, wie sie innerhalb einer bestimmten Population bestehen und von der Wissenschaft, insbesondere der Sozialpsychologie gegebenenfalls als solche registriert und interpretiert werden: als Klischeevorstellungen, Stereotype, Vorurteile und dergleichen. Es geht hier - bei der Frage nach dem Typischen im Allgemeinen also nicht darum, dass bestimmte Merkmale, Eigenschaften, Verhaltensweisen typisch deutsch sind, sondern dass sie als typisch deutsch gelten; wobei es wiederum eine entscheidende Rolle spielt, wer diese Merkmale, Eigenschaften, Verhaltensweisen usw. für typisch hält - in der Terminologie Hofstätters (1966) um die Frage: ob es sich bei diesem »typisch soundso « um ein »Autostereotyp« oder ein »Heterostereotyp « handelt, aber auch um die Frage, ob und worin sich die verschiedenen Heterostereotypen voneinander unterscheiden. Zusammenfassend: Die Sozialpsychologie - im Sinne Hofstätters - befasst sich bei ihren Objekten in der Regel nicht mit der Frage, wie diese Objekte beschaffen sind, sondern damit, welche Art von Beschaffenheit ihnen zugeschrieben wird.

Zurück zu Bausinger, der in seinen Überlegungen zum Begriff des Typischen - bedauernd, wie mir scheint - feststellt: »Das Typische lässt sich nicht durch genaue Zählungen oder Messungen ermitteln« (Bausinger 2000: 21). Ich stimme zu, aber nicht bedauernd. Ich weiß natürlich, dass das Zählen und Messen zu den bevorzugten Instrumenten empirisch-sozialwissenschaftlicher Forschung gehört. Aber es gibt auch noch andere Instrumente. In der Musikwissenschaft beispielsweise gibt es das mittlerweile zugegebe- 
nermaßen zunehmend an den Rand geratene, jedoch nach wie vor außerordentlich wichtige Werkzeug der musikalischen Analyse, in unserem Fall besonders der Stilanalyse. Ich komme darauf zurück.

Die Stereotypenforschung der 1950er und 1960er Jahre pflegte - im Sinne Bausingers »Zählen und Messen« - eine streng empirische Herangehensweise an ihren Gegenstand. Das wichtigste Messinstrument war das von C.E. Osgood und seinen Mitarbeitern entwickelte und in der Arbeit The Measurement of Meaning (1957) vorgestellte »Semantische Differential«, in Deutschland zumeist als Polaritätsprofil bezeichnet, eine Methode, die den Bedeutungsgehalt von Objekten, Begriffen, Personengruppen usw. mit Hilfe von verbalen Zuordnungen, z.B. von gegensätzlichen Adjektivpaaren, zu bestimmen und mittels relativ komplizierter korrelationsstatistischer Verfahren wie der Faktorenanalyse und später der Clusteranalyse zu analysieren. Der Stereotypenforschung ging es dabei nicht darum festzustellen, wie der jeweilige Gegenstand tatsächlich beschaffen war, sondern darum, was er für bestimmte Personengruppen bedeutete, nicht darum, seine objektiv vorhandenen Eigenschaften zu ermitteln, sondern das Bild zu erfassen, das sich verschiedene Gruppen unterschiedlichen Geschlechts, unterschiedlicher $\mathrm{Na}$ tionalität, unterschiedlicher Berufe - oder unterschiedlicher was auch immer - von ihrem jeweiligen Untersuchungsgegenstand machten.

Am Rande sei vermerkt: Ich persönlich interessiere mich für derartige Untersuchungen, die auf der Analyse von Durchschnitts- oder Mittelwerten basieren, schon sehr lange nicht mehr. Und seit ich 1974 in Forschung in der Musikerziehung einen Artikel unter dem Titel »Über den Fetischcharakter des Mittelwerts « veröffentlichte (vgl. auch Jost 1973) und dafür erheblichen Gegenwind von Seiten der Fraktion der Strichlisten-Auszähler bekam, habe ich mich vom Terrain des Naiven Empirismus sorgfältig ferngehalten.

Dieser Art von Erkenntnisgewinn auf der Basis von empirischen Methoden der Sozialpsychologie, Psychologie oder der Sozialforschung, der es weniger darum geht festzustellen, was etwas ist, als darum, wofür etwas gehalten wird, eine Form des Erkenntnisgewinns also, die letztlich eine enge Verwandte der populären Markt- und Meinungsforschung bildet, stellen die Kunst- und Musikwissenschaften nun allerdings Methoden gegenüber, die primär nicht darauf abzielen herauszufinden, wofür etwas gehalten wird, sondern was etwas ist. Es handelt sich dabei um hermeneutische Ansätze, die sich allerdings nicht allein oder vorrangig auf Introspektion oder einsame Denkprozesse stützen, sondern die von einer detaillierten, fachspezifischen Analyse des betreffenden Gegenstandes ausgehen. Eine derartige Methode liefern in unserem Fall (ich meine im Fall der Musikwissenschaft) 
die verschiedenen Verfahren der »Musikalischen Analyse « und insbesondere der Stilanalyse.

Wenn es mir darum geht zu erfahren, was das »typisch Deutsche « an einer bestimmten Musik, d.h. einem bestimmten musikalischen Genre, einem bestimmten Zeit- oder Epochenstil usw. ist, bin ich darauf angewiesen, auf der Basis einer möglichst großen Stichprobe von Musikbeispielen und ausgehend von möglichst detaillierten Analysen der strukturellen Beschaffenheit dieser Musik, ihrer wichtigsten Gestaltungsprinzipien, ihrer Ausdrucksmittel usw. meine Aussagen zu treffen. Aber das reicht noch keineswegs aus, denn um dem »typisch Deutschen « an meinem Untersuchungsgegenstand auf die Schliche zu kommen, bedarf es schließlich unbedingt erst einmal des Vergleiches mit den entsprechenden und vergleichbaren musikalischen Genres oder Zeit- oder Epochenstilen anderer Völker, Kulturen, Nationen, Ethnien. Was wiederum die Analyse und die daraus resultierende Vertrautheit mit den entsprechenden musikalischen Hervorbringungen anderer vergleichbarer Völker, Kulturen, Nationen, Ethnien usw. voraussetzt.

Die Methode der Stilanalyse ist, wie ich aus langjähriger Erfahrung und der Arbeit an sehr verschiedenen Arten von Musik weiß, eine sehr mühevolle Angelegenheit. Was ist das überhaupt, ein Stil? Ist das auch eine Zuschreibung (von außen gewissermaßen)? Oder ist das ein Faktum, ermittelt auf der Basis intensiver analytischer Bemühungen? Oder ist es ein Konstrukt, basierend auf der Bündelung der durch derartige analytische Bemühungen gewonnenen Erkenntnisse? Mit letzterem kommen wir der Sache - wie ich meine - schon wesentlich näher.

Während der Arbeit an meiner Sozialgeschichte des Jazz in den USA (1982) und der damit verbundenen Auseinandersetzung mit dem Stilbegriff bin ich fast zwangsläufig auf die Schriften des großen Soziologen Max Weber (1864-1920) gestoßen. Ein Schlüsselbegriff in den Überlegungen Webers ist der des »Idealtypus«. Der »Idealtypus«, den Weber später übrigens durch den des »konstruierten Typus « ersetzte, um ihn ausdrücklich von einem idealen (vorbildlichen) Typus im normativen Sinne zu unterscheiden, der Idealtypus bezeichnet - nach Weber - ein Konstruktion, die dazu dient, »einen Komplex von Zusammenhängen in der geschichtlichen Wirklichkeit [...] begrifflich zu einem Ganzen zusammenzuschließen« und damit eine unübersichtliche Realität überhaupt erst beschreibbar zu machen. Ein Idealtypus »wird gewonnen durch einseitige Steigerung eines oder einiger Gesichtspunkte und durch Zusammenschluss einer Fülle von diffus und diskret, hier mehr, dort weniger, stellenweise gar nicht, vorhandener Einzelerscheinungen, die sich jenen einseitig herausgehobenen Gesichtspunkten fügen - zu einem in sich einheitlichen Gedankengebilde« (Weber 1968: 68). 
Die Stilbereiche des Jazz, Swing, Bebop, Free Jazz usw. sind solche Gedankengebilde, die in der Totalität der sie konstituierenden Merkmale nirgendwo real auffindbar sind und die dennoch auf Reales sich beziehen. Musikalische Stilbegriffe bezeichnen theoretische Konstruktionen. Es sind Gedankengebilde, die dazu dienen, einen Komplex von musikalischen Zusammenhängen begrifflich zu einem Ganzen zusammenzuschließen und damit eine unübersichtliche Realität überhaupt erst beschreibbar und damit verstehbar zu machen. Man darf sich durch sie nur nicht zum Schubladendenken verleiten lassen, darf sie nicht für etwas Starres, ein für allemal Fix und Fertiges halten, sondern muss ihre Dynamik erkennen. Wie stets in solchen Fällen, kann es auch bei dem Versuch einer Herausarbeitung des Deutschen oder Britischen oder Französischen im europäischen Jazz der 1970er Jahre also nur darum gehen, einige Merkmale herauszudestillieren, die als einigermaßen typisch angesehen werden können; musikalische Charakteristika, die einerseits bis zu einem gewissen Grade zu verallgemeinern sind, sich also nicht nur auf einen oder einige wenige Musiker oder Gruppen beziehen, und die andererseits dazu geeignet erscheinen, diesen als deutsch, britisch oder französisch identifizierten Jazz gegenüber dem anderer Länder unterscheidbar zu machen.

Das von europäischen Musikern während der 1960er Jahre entwickelte Konzept einer free music, aus welchem bezeichnenderweise der Begriff Jazz mitunter getilgt war, zielte darauf $a b$, dass man sich frei entfalten konnte, ohne Rücksicht auf tradierte Normen und überkommene Klangvorstellungen. Die Frage stellte sich alsbald: Gibt es eigentlich so etwas wie einen Aufbruch in die totale Freiheit, in einen voraussetzungslosen Individualismus? Ziemlich rasch stellte sich da heraus, dass man offenbar nicht mit einem Ruck seine ganze Geschichte abschütteln kann, seine Erfahrungen auf einen Schlag tilgen, so wie man einen Vertrag zerreißt, ein Denkmal zertrümmert oder einen Flipperautomaten auf Null stellt: Tilt! Denn die Aufkündigung des Abhängigkeitsverhältnisses gegenüber den US-amerikanischen Vaterfiguren bedeutete ja nicht zugleich auch die Auslöschung der eigenen jazzmusikalischen Erfahrungen, welche die meisten der betreffenden europäischen Musiker bis dahin gemacht hatten, und sie bedeutete vor allem auch nicht einen Rückzug aus der eigenen, historisch gewachsenen kulturellen Identität, sondern provozierte - im Gegenteil - eine stärkere Reflexion derselben (vgl. hierzu Jost 1987: 11ff.).

Aber gab es so etwas überhaupt, eine europäische kulturelle Identität? War nicht die europäische Kulturlandschaft alles andere als gleichförmig, sondern außerordentlich vielfältig? Und war nicht das Ausmaß, in welchem einzelne Musiker an den diversen Ausdrucksformen europäischer Kultur teil- 
hatten, alles andere als einheitlich, sondern - bedingt durch soziale Voraussetzungen und kulturelles Umfeld - sehr unterschiedlich? Es lag also durchaus nahe, dass sich in der Folge der Negation des US-amerikanischen JazzIdioms durch europäische Musiker nicht etwa sogleich eine überregionale europäische Jazzsprache herauskristallisierte, sondern eine Vielzahl verschiedenartiger Dialekte, dass es zu ganz unterschiedlichen Gestaltungsweisen und Ausdrucksmitteln kam, die - zumindest zum Teil - mit den unterschiedlichen kulturellen Identitäten der betreffenden Musiker in Zusammenhang standen.

So genannte »Nationalstile « in den Künsten und der Literatur sind seit der Renaissance nichts Außergewöhnliches. Und wenngleich das 20. Jahrhundert mit seinem medienbedingten kulturellen Internationalismus die meisten Differenzen einebnete, so sind doch bis heute - beispielsweise in den Bereichen der Literatur und der zeitgenössischen akademischen Musik gewisse nationale Eigentümlichkeiten auszumachen. Dies hat nichts mit $\mathrm{Na}$ tionalismus oder Chauvinismus im politischen Sinne zu tun, sondern ist durch die unterschiedliche Geschichte einzelner Völker und Nationen (die ja stets ihre Kulturgeschichte einschließt) bedingt.

Die Frage, ob auch im zeitgenössischen Jazz derartige unterschiedliche nationale Tendenzen auffindbar sind, wäre noch bis in die 1960er Jahre hinein kaum jemandem in den Sinn gekommen. Jazz wurde stets als musikalische Weltsprache US-amerikanischer Herkunft aufgefasst und als solche mit Selbstverständlichkeit propagiert. Mit dem Einzug der freien Spielformen des Jazz in Europa jedoch hatte diese »Weltsprache« Jazz ihre Allgemeinverbindlichkeit verloren.

Was sich in dem Bruch zwischen europäischer und amerikanischer Free Jazz-Ästhetik vergleichsweise krass andeutete, galt - wenn auch mit weitaus geringerer Trennschärfe - für bestimmte regionale Spielarten des europäischen Free Jazz. Seit Anfang der 1970er Jahre etwa war es üblich, dass unter Musikern und Insider-Publikum von den Holländern oder den Engländern gesprochen wurde, wobei die nationale oder geographische Differenzierung sich in der Regel nicht nur auf bestimmte Gruppen von Musikern bezog, sondern zugleich eine bestimmte Auffassung von Musik einschloss. Und so begann man alsbald von einer spezifisch britischen Art der Klangmaterialforschung zu sprechen, von einer besonderen Form niederländischer Humormusik oder einer typisch »germanisch-teutonischen « Art der Energieproduktion. All dies waren natürlich zunächst einmal Klischees, stereotype Vereinfachungen, die der jazzmusikalischen Wirklichkeit in den einzelnen Ländern niemals ganz gerecht wurden. Aber immerhin: derartige Klischees, so eindimensional und grob sie auch erscheinen mochten, waren darum 
doch keineswegs völlig aus der Luft gegriffen, sondern bezeichneten - wie unscharf auch immer - eine real bestehende regionale oder nationale stilistische Differenzierung, bezeichneten also (gleich, ob man den Begriff nun mag oder nicht) so etwas wie jazzmusikalische National- oder Regionalstile.

Wie die meisten Klischees und stereotypen Begriffsbildungen waren auch die musikbezogenen also einerseits unscharf, andererseits aber durch Realität hervorgerufen. Sie waren geprägt durch unterschiedliche gesellschaftliche und kulturelle Verhältnisse, beziehungsweise durch eine unterschiedliche Wahrnehmung und Verarbeitung dieser Verhältnisse von Seiten der betreffenden Musiker. Offensichtlich bildeten sich derartige regionale, beziehungsweise nationale Besonderheiten vor allem dort aus, wo von Anfang an eine vergleichsweise umfangreiche und aktive Free-Szene bestand. Und dies war in Westeuropa vor allem in Großbritannien, den Niederlanden, der Bundesrepublik, Frankreich und - mit geringer Zeitverzögerung - in der DDR der Fall.

\section{Exkurs}

Dass eine derartige stilistische Differenzierung, die verschiedene national oder regional gebundene jazzmusikalischen Idiome oder Stile unterscheidbar macht, nicht nur lediglich das Ergebnis musikwissenschaftlicher Analyse und Reflexion war, sondern im Alltag der Jazzszene tatsächlich ihre Entsprechung fand, möchte Ihnen anhand einer kleinen Anekdote nahe bringen, einer Anekdote, die nicht erfunden ist, sondern die das Leben schrieb. Ich durfte sie während eines internationalen Jazzfestivals in Rom erleben, zu dem man mich im Herbst 1992 eingeladen hatte. Dieses Festival, das von einer italienischen Jazzmusikerkooperative organisiert worden war, war ein Festival besonderer Art. Es hieß »Controindicazione«, ein medizinischer Terminus, der so viel bedeutet wie »Gegenanzeige«, »Gegenmittel«, ein Begriff, der in diesem Rahmen offensichtlich den herkömmlichen Festivalrummel der damaligen Jazzszene im Visier hatte, gegen den man sich wandte.

Das Grundprinzip dieser dreitägigen bzw. dreinächtlichen Veranstaltung bestand darin, dass keine, oder so gut wie keine festen Gruppen dort auftraten, sondern ausschließlich ad hoc und vor Ort zusammengestellte Ensembles, wobei Wert darauf gelegt wurde, dass möglichst Musiker zusammengespannt wurden, die einander nicht kannten, vielleicht voneinander gehört, aber niemals miteinander gespielt hatten. Und so kam es, dass ich eines Abends mit zwei freundlichen italienischen Kollegen konfrontiert wurde, die ich niemals vorher gesehen hatte: dem Perkussionisten Mauro Orselli aus Rom und dem Multi-Instrumentalisten (Saxophon, Klarinette, Klavier und 
Violine) Renato Geremia aus Venedig. Zu den Selbstverständlichkeiten dieser Aufführungspraxis gehörte auch, dass man vorher keine nennenswerten Absprachen traf, geschweige denn miteinander probte. Meine beiden italienische Mitspieler teilten mir lediglich in ihrem unnachahmlichen Italo-English mit: »We« (d.h. die beiden Italiener) »start at nine-thirty, and you join us ten minutes later.«

Das war's! Und dann beging ich einen Tabubruch und sagte - sehr freundlich aber bestimmt - »Please don't play British«. Und beide brachen vor Lachen fast zusammen. Was war passiert? Es hatte vor uns eine Gruppe von sehr jungen italienischen Musikern gespielt, die in ihren freien Improvisationen alle Merkmale jener als »typisch britisch « qualifizierten Musik vereinigten: Reine Klangforschung, quasi asketisch, d.h. unter Verzicht auf als solche erkennbaren Emotionen, eine Musik, die von einer Vielzahl von Verbotsschildern umstellt war: No melody, no harmony, no time - fragil, aber nach zehn Minuten ein wenig langweilig.

»Don't play British«, »British« - hier offenbar ein Begriff, der auch auf der italienischen Jazzszene Signalcharakter hatte: »Du sagst >Don't play British <, dann weiß ich schon, was Du damit meinst. - Später im Foyer an der Bar hörte ich dann immer wieder: »Did you get it? Joste says: Don't play Britishe«, worauf unweigerlich ein schallendes Gelächter zu vernehmen war.

Was lernen wir daraus? Es gibt offenbar Formen der musikalischen Praxis, musikalische Merkmale, Ausdrucksmittel, Gestaltungsprinzipien, Idiome, die sich in der Tat als typisch für bestimmte Regionen, Länder, Nationen usw. identifizieren lassen, die im kollektiven Bewusstsein bestimmter Populationen - möglicherweise unbewusst oder zumindest unreflektiert - als Selbstverständlichkeiten vorhanden sind. Ich könnte mir vorstellen, dass die beiden italienischen Kollegen Geremia und Orselli, wenn ich ihnen gesagt hätte »Don't play Wuppertal«, das für teutonisches Powerplay steht, ebenso schnell verstanden hätten, worum es geht.

Ich möchte nun versuchen, anhand von drei Beispielen - Westdeutschland, Großbritannien und Frankreich - zumindest umrisshaft zu verdeutlichen, worin derartige stilistische Differenzierungen bestanden. Beginnen wir - vor Ort - mit der Bundesrepublik Deutschland. Die Free Jazz-Szene Westdeutschlands Ende der 1960er, Anfang der 1970er Jahre war geprägt durch die Musiker der so genannten »Achse Berlin-Wuppertal«; Berlin, weil dort zu Ende der 1960er Jahre die »Free Music Production« (FMP) ihre Arbeit aufnahm, eine außerordentlich emsige Musiker-Kooperative, zu deren Aktivitäten die Gründung eines später weltweit bekannten Schallplatten-Labels 
und die Durchführung zahlreicher bedeutender Konzerte und Festivals gehörten; Wuppertal, weil dort einige der wichtigsten mit der mit der FMP assoziierten Protagonisten des neuen Jazz stationiert waren: Ich erwähne hier nur Peter Brötzmann und Peter Kowald.

Zu den hervorstechenden Merkmalen der Musik der Gruppen im Umkreis der FMP gehörten die folgenden (vgl. Jost 1987: 113ff.):

(1) Kompositorische Maßnahmen blieben im Allgemeinen auf ein Minimum beschränkt. Das heißt, im Großen und Ganzen war die Musik a-thematisch, wobei es von Fall zu Fall lediglich riffartig eingesetzte, bisweilen nur in ihrer Richtung fixierte Einwürfe und Signale gab. Daneben bestand eine gewisse Neigung zu verfremdeten Zitaten, wie in Peter Brötzmanns »Lollopalooza «2 ${ }^{2}$, wo die Nationalhymnen der Bundesrepublik und Großbritanniens auf bewusst chaotische Weise einander verflochten wurden.

(2) Die eindeutig fixierbare Tonhöhe als konstitutives Element musikalischer Organisation wurde weitgehend aufgegeben zugunsten von instabilen Klangflächen. Strukturelle Differenzierung vollzog sich vor allem durch die kollektive Variation der Parameter Klanghöhe, -dichte und -stärke.

(3) Entwicklungsprozesse wurden mit einiger Zwangsläufigkeit an die Grenzen des Durchhörbaren geführt, das heißt, in Bereiche, in denen einzelne musikalische Ereignisse nicht mehr identifizierbar wurden, sondern ein diffus-intensives Ganzes bildeten. Nicht selten trug dazu die Instrumentation einiges bei, die besonders in den Brötzmann-Gruppen extrem homogen und tieflagig war.

(4) Die Einstellung zur Zeit war im Allgemeinen als rastlos zu bezeichnen. Auch dort, wo sich aufgrund abnehmender Klangdichte und -stärke offenere Strukturen etablierten, blieb der Puls meistens sehr schnell. In Manfred Schoofs European Echoes (FMP 0010, 1969), liegt er fast durchgehend bei 300 bpm. Diese letztere Tendenz zur Hektik, verbunden mit einer rhythmischen Grundhaltung, in der Swing im traditionellen Sinne kaum noch spürbar wurde, war generell im gesamten europäischen Free Jazz der späten 1960er und frühen 1970er Jahre vorhanden, für die Musik der Orchester im Umkreis der FMP gehörte sie zu den charakteristischen, also typischen Merkmalen.

Eine der wichtigsten Kernzellen der britischen Jazz-Avantgarde war seit Mitte der 1960er Jahre der von dem Perkussionisten John Stevens initiierte Little Theatre Club, eine Art musikalischer Werkstatt, in der - ohne Rücksicht auf das ohnehin spärliche Publikum - mit einer Intensität und Konse-

21969 eingespielt auf Alexander von Schlippenbachs The Living Music (FMP 0100). 
quenz experimentiert wurde, die in der Geschichte des Jazz vielleicht nur in den in Harlem stattfindenden Monroe- und Minton-Sessions beim Anbruch der Bebop-Ära eine Parallele findet. Die zentrale Formation innerhalb der ständig wechselnden Besetzungen, die im Little Theatre Club auftraten, bildete das von John Stevens gemeinsam mit dem Saxophonisten Trevor Watts gegründete Spontaneous Music Ensemble (SME), eine Formation, in der - für mehr oder minder lange Zeit - nahezu jeder Musiker der Londoner Avantgarde-Szene mitwirkte (vgl. Jost 1987: 52ff.).

Die Musiker der britischen Free Jazz-Szene gelangten während ihrer experimentellen Phase auf eine Abstraktionsebene, die Melodik und Harmonik nicht nur in Frage stellte, sondern gleichsam tabuisierte. Geräuschhaftigkeit bildete dabei keine Alternative mehr, sondern wurde bestimmendes Gestaltungsprinzip. Was man im Rahmen der sogenannten musique concrète per Tonbandcollage erreichte, produzierten britische Musiker der KlangforscherSzene live. Instrumentenspezifische Charakteristika wurden konsequent negiert oder ins Gegenteil verkehrt: während Saxophonisten perkussiv knatternde, geräuschhafte Rhythmuspatterns produzierten, sorgten Schlagzeuger für klangfarbliche Vielfalt, Bassisten strichen zwitschernd im Falsett, Gitarristen (besonders Derek Bailey) produzierten ohne elektro-akustische Hilfsmittel Sounds, die man anderenorts nur aus den aufwendigen Gerätschaften von Studios für elektronische Musik zu hören bekam.

Am konsequentesten in der Abkehr von den traditionellen Gestaltungsmitteln des Jazz war ein Quartett namens Music Improvisation Company mit Derek Bailey (Gitarre), Hugh Davis (Live-Elektronik), Jamie Muir (Perkussion) und Evan Parker (Sopransaxophon). Die vier Musiker der Company distanzierten sich vom Jazz ebenso wie von der neuen E-Musik: Vom Jazz vor allem durch ihre Einstellung zum Rhythmus, aus dem alles verbannt war, das noch so entfernt mit der rhythmischen Qualität des Swingens assoziierbar war. Zur Neuen E-Musik gingen sie auf Distanz vor allem aufgrund ihres programmatischen Verzichts auf Planung. Komposition war - von Ausnahmen abgesehen - unerwünscht, vorherige Absprachen ebenso verpönt wie anschließendes Analysieren: music is now hieß die Devise.

Der britische Free Jazz der 1960er und frühen 1970er Jahre neigte in seiner emotionalen Grundhaltung - anders als die Musik der FMP-Leute weniger zur Ekstase und zur Energieproduktion als vielmehr zur Askese und zeigte Tendenzen zur abendländischen so genannten E-Avantgarde, ohne sich dieser jedoch so weit anzupassen, dass ihm dabei die Spielfreude verloren ging. 
Ich spreche davon, dass es innerhalb des Stilkonglomerates Free Jazz in Europa verschiedene, regional (oder national) gebundene Dialekte gibt. Ich spreche von einer »teutonischen Form der Energieproduktion« und von »britischer Klangforschung «. Stellt sich die Frage: was ist bzw. war das Französische im zeitgenössischen Jazz Frankreichs?

Die Frage ist nicht so leicht zu beantworten, denn dieser neue Jazz in Frankreich erwies sich in stilistischer Hinsicht als außerordentlich vielfältig und durch ein prägnantes Etikett kaum auf den Nenner zu bringen. Hinzu kommt, dass die französische Jazzszene wesentlich länger und gründlicher als andere in Europa unter dem dominierenden Einfluss der US-Amerikaner stand, dass somit regionale oder nationale Extratouren schon aufgrund der ständigen Präsenz US-amerikanischer Musiker auf dieser Szene im Hintergrund blieben. Zu Ende der 1960er Jahre begann sich daran einiges zu ändern. Das Französische im französischen Jazz trat deutlicher zu Tage.

Wie stets in solchen Fällen, kann es auch bei dem Versuch einer Herausarbeitung des Französischen im französischen Jazz der 1970er Jahre nur darum gehen, einige Merkmale herauszudestillieren, die (sagen wir es mal vorsichtig) als einigermaßen typisch angesehen werden können, musikalische Charakteristika, die einerseits bis zu einem gewissen Grade zu verallgemeinern sind, sich also nicht nur auf einen oder einige wenige Musiker beziehen, und die andererseits dazu geeignet erscheinen, diesen als französisch identifizierten Jazz gegenüber dem anderer Länder unterscheidbar zu machen.

Drei Kriterien wären zu nennen, versuchsweise und im Bewusstsein des analytischen Glatteises, auf das wir uns begeben: erstens eine spezifische Einstellung zur Melodik und - zum Teil damit verbunden - zur eigenen musikalischen Folklore und Chansontradition; zweitens eine stilistische Mehrgleisigkeit, in der das Schauen über den Zaun der kleinen Jazzwelt zum Prinzip erhoben wird; und drittens eine bestimmte Art und Weise, mit Musik umzugehen, sie in Szene zu setzen. Gehen wir ins Detail (vgl. Jost 2012: 267-270):

Erstens: Eines der hervorstechenden Merkmale des freien Jazz in Frankreich während der 1970er Jahre lag im Festhalten an einer quasi vokalen Art von Melodik, einer Melodik, die auch im freiesten Kontext noch eine gewisse Nähe zum Sanglichen bewahrte, die sich also nicht in Klänge aufsplitterte oder zu flächenhaften Gebilden deformierte, sondern die - im weitesten Sinne - eine instrumentale Übersetzung vokaler Melodieerfindung darstellte. Natürlich spielten auch französische Saxophonisten und Blechbläser mit abstrakten Sounds, mit a-melodischen Mehrklängen oder energiebetonten 
Schreiphrasen. Jedoch ihre musikalische Grundhaltung war zumeist melodieorientiert. Sie blieben, bei allen Freiheiten, die sie sich nahmen - in erster Linie Melodiker, »Sänger «, und erst in zweiter Linie Klangexperimentatoren. Dabei bestand bei zahlreichen Musikern der französischen Free-Szene eine auffallend starke Affinität zur Volksmusik, was sich nun allerdings keineswegs in eine griffige Stilkategorie von Free Folk übersetzen ließe. Vielmehr handelte es sich bei dieser folklore imaginaire um eine mehr oder minder vage ästhetische Orientierung, welche die liebevolle, spielerische oder auch ironisierende Verarbeitung folkloristischer Muster einschloss, ohne dabei in die Falle einer romantisch verklärten Vergangenheitsbewältigung zu tappen. Halten wir fest: die französischen Free Jazz-Spieler waren und sind (zum überwiegenden Teil) Melodiker - freie Melodiker.

Zweitens: Ein weiteres für die französische Free-Szene charakteristisches Merkmal bestand in einem Phänomen, das man als »musikalisches Grenzgängertum « bezeichnen könnte. Es gab in Frankreich vergleichsweise viele Musiker, die sich nicht auf einen Stilbereich, ja nicht einmal auf ein einziges musikalisches Genre wie Jazz, Neue Musik oder Popularmusik festlegen ließen, sondern für die das Wandern zwischen den musikalischen Welten selbstverständlich war. Viele dieser Musiker hatten nicht nur auf der Jazzszene ihre Meriten, sondern besaßen auch als hervorragende Interpreten Neuer E-Musik einen guten Namen.

Dieses musikalische Grenzgängertum französischer Musiker beschränkte sich allerdings nicht auf das Überschreiten von Grenzen zwischen Jazz und Neuer Musik, sondern schloss vielfach auch mehr oder minder reibungslose Übergänge zur sogenannten Unterhaltungsmusik ein. Dies mochte zum einen daran liegen, dass die Grenzen zwischen hoher Kunst und Unterhaltung in Frankreich ohnehin niemals so streng bewacht wurden wie anderswo; darüber hinaus aber dürfte auch das im großen und ganzen recht hohe Niveau französischer Unterhaltungsmusik, speziell in Gestalt des Chansons, eine Rolle gespielt haben.

Beides, die Fähigkeit, mit der Musik der E-Avantgarde kompetent umzugehen und die relativ unverkrampfte Haltung gegenüber der populären Musik ihres Landes, fand - gefiltert und gesiebt - seinen Niederschlag in der Musik der französischen Jazz-Avantgarde, sei es, dass man zu formalen Problemlösungen gelangte, wie sie sich in der Neuen E-Musik andeuteten, sei es, dass man - offensichtlich ohne gravierende ästhetische Skrupel mit den simpelsten U-Musik-Fragmenten hantierte, denen Mangel an Tiefgang und Kunstfertigkeit vorzuwerfen unsinnig wäre, denn sie beanspruchten weder das eine noch das andere. 
Drittens: Aus den erwähnten Beispielen dürfte deutlich geworden sein, dass von dem französischen Avantgardejazz im Sinne eines eindeutigen Stilbegriffs ebenso wenig die Rede sein konnte wie vom deutschen, britischen oder niederländischen. Wohl aber sind einige charakteristische Züge auszumachen, die dem neuen Jazz in Frankreich ein relativ hohes Maß an Eigenständigkeit verliehen, die ihn als solchen identifizierbar machten. Zu den bislang genannten Eigenarten kam nun allerdings eine weitere, die mir besonders evident erscheint, auch wenn sich gerade sie nur mühsam in Worte fassen lässt. Den dafür gewählten Begriff der »Spielhaltung « borge ich mir von Bert Noglik, der inn in seinen »Werkstattnotizen« als zentrale Kategorie zur Sprache brachte. »Spielhaltung « bezeichnet - nach Noglik (1981: 475) eine Art der musikalischen Einstellung, aber auch eine spezifische Art und Weise, mit Musik umzugehen, sie in Szene zu setzen. Die Spielhaltung der französischen Avantgardemusiker der 1970er Jahre ist schwer zu verbalisieren; man sollte sie erlebt haben. Es war eine Haltung, der eine gewisse Leichtigkeit innewohnte, von der gleichsam die Aufforderung ausging, nun mal nicht alles so todernst zu nehmen. Es mag sein, dass diese Haltung, die mir nirgends so ausgeprägt erschien wie bei den Franzosen, zusammenhing mit der vorher beschriebenen stilistischen Mehrgleisigkeit, mit ihrer Neigung, über den eigenen jazzmusikalischen Zaun zu schauen und das gesamte musikalische Universum - oder jedenfalls doch große Teile davon - als Spielmaterial ins Auge zu fassen.

Nun hatte ich allerdings in der Überschrift meines Beitrages in Aussicht gestellt, ich wolle versuchen, den Begriff des Typischen auf sinnvolle Weise durch einen anderen zu ersetzen. Ich würde vorschlagen: Spezifisch, wobei es mir allerdings weniger auf das Wort selbst ankommt (man könnte auch sagen charakteristisch, unverwechselbar, symptomatisch usw.), sondern vielmehr darauf, auf welcher Grundlage eine derartige Aussage getroffen wird - aufgrund einer Zuschreibung durch den Hörer, beziehungsweise der journalistischen Publizistik? Oder auf Grund einer musikwissenschaftlich fundierten Analyse, die nicht darauf abzielt herauszufinden, ob ein Merkmal als spezifisch für einen bestimmten Untersuchungsgegenstand gilt, sondern ob es spezifisch für diesen Gegenstand ist.

Daraus folgt mein Appell an meine jüngeren Kollegen aus der musikologischen Zunft: Kümmert Euch mehr um die Musik selbst als um das Reden über Musik. Das Letztere erfreut sich in den letzten Jahren zwar allenthalben wachsender Beliebtheit, sollte meines Erachtens jedoch nicht im Zentrum unserer Arbeit stehen. 


\title{
Literatur
}

Bausinger, Hermann (2000). Typisch deutsch. Wie deutsch sind die Deutschen? München: C.H. Beck.

Dorn, Thea / Wagner, Richard (2011). Die deutsche Seele. München: Knaus.

Durand, Béatrice (2004). Die Legende vom typisch Deutschen. Eine Kultur im Spiegel der Franzosen. Leipzig: Militzke.

Gelfert, Hans-Dieter (2005). Was ist deutsch? Wie die Deutschen wurden, was sie sind. München: C.H. Beck.

Hofstätter, Peter R. (1966). Einführung in die Sozialpsychologie. Stuttgart: Kröner.

Jost, Ekkehard (1973). »Anmerkungen zur Rolle der Versuchsperson in der experimentellen Musikpsychologie.« In: International Review of the Aesthetics and Sociology of Music 4, H. 2, S. 229-244.

Jost, Ekkehard (1974). »Über den Fetischcharakter des Mittelwerts. Methodische Probleme der experimentellen Musikpsychologie.« In: Forschung in der Musikerziehung 1974. Hg. v. Bernhard Binkowski, Siegmund Helms und Hans-Peter Reinecke. Mainz: Schott, S. 95-105.

Jost, Ekkehard (1982). Sozialgeschichte des Jazz in den USA. Frankfurt/M.: Fischer. Jost, Ekkehard (1987). Europas Jazz 1960-80. Frankfurt/M.: Fischer.

Jost, Ekkehard (2012). Jazzgeschichten aus Europa. Hofheim: Wolke.

Noglik, Bert (1981). »Werkstattnotizen.« In: Jazzwerkstatt International. Hg. v. dems. Berlin (DDR): Verlag Neue Musik, S. 475-480.

Osgood, Charles Egerton / Suci, George J. / Tannenbaum, Percy H. (1957). The Measurement of Meaning. Urbana, IL: University of Illinois Press.

Vannuccini, Vanna / Predazzi, Francesca (2013). Feierabend. Eine Reise in die deutsche Seele. München: Riemann.

Weber, Max (1968). Methodologische Schriften. Studienausgabe. Frankfurt/M.: S. Fischer.

\begin{abstract}
Word formations like »typically...« belong to the same semantic field as clichés, prejudices and stereotypes and therefore are within the responsibility of social psychology in the first place, which is - if at all - hard to approach with music analytical methods but remains the realm of music criticism or the feuilleton and other speculative fields of thought. Consequently, I study my topic first from a methodological and social psychological standpoint. I then show with the help of the example of the so called »European Jazz Emancipation« of the 1960s, how and why typical characteristics emerge in music and especially in jazz, which may justify to a certain extend the (cautious) use of the term typical.
\end{abstract}

